\title{
Simulation of Piezoelectric Stack Actuators for Loss Component
}

\author{
Mark Christian P. Espinosa, Paola N. Calub, Rodney B. Javier* and Sheryl Dinglasan Fenol
}

College of Engineering and Information Technology, Cavite State University, Indang, Cavite, Philippines

Received 26 May 2021; Accepted 22 September 2021

\begin{abstract}
Piezoelectric actuators (PEA) are widely utilized now for ultra-precision positioning and actuation in the micro/nanotechnology manufacturing industry. To ensure that these devices perform efficiently and to improve the system and prevent unnecessary inaccuracies it may trigger, it is better to understand its loss phenomenology. Piezoelectric devices have three losses: dielectric, mechanical, and piezoelectric. In this study, behaviour of PEA was simulated using COMSOL Multiphysics to characterize each loss and determine its effect on mechanical response, electrical impedance and percentage loss. The quality factor of the PEA was also calculated with different combined loss. The simulation study showed that each loss has its own influence on the said constituents especially over the frequency sweep. It was also proved that resonant frequency is invariant to the losses, thus, making it an extensive property of the PZT material.
\end{abstract}

Keywords: Piezoelectric Actuator, Loss Phenomenology, COMSOL Multiphysics, Quality Factor, Actuators

\section{Introduction}

The extensive study of piezoelectric actuators (PEA) for micro-positioning applications made a lot a progress through some times due to six main advantages given by Li \& Chen (2013) [1] and Chi \& Xi (2014) [2] such as not omission of a rotating mechanism, ultrahigh precision positioning, instant response, very large output force, lower power dissipation and design aesthetic. It contributed a lot in enabling micro/nanotechnology and ultra-precision manufacturing systems. It is then necessary to enhance the efficiency and performance of this component to increase the mechanical factor and clarify its loss phenomenology [3].

Hysteresis is an innate nonlinear property of piezoelectric devices that influence its control performance and efficiency. Displacement of PEAs depends on not only the current control inputs but also the historical inputs because of the existence of hysteresis [4-5]. According to Zhuang et al. (2014) [6], hysteresis losses in piezoelectrics are considered to have three types in general: dielectric, mechanical (elastic), and piezoelectric losses where they can be classified into intensive or extensive factor upon the boundary conditions $[2$, $6,7,8]$

Several hysteresis models, as indispensable parts in improving the performance of PEA, have been proposed to describe the hysteresis effect [4, 9]. Chi \& Xi (2014) [2] categorizes these models into two types: physics-based models and phenomenological models. Physics-based models utilize the relationship of energy, the displacement and etc. such as Zhang \& Damjanovic (2020) [5] described using the Quasi-Rayleigh model while phenomenological models directly uses mathematical models starting from the characteristics of the hysteresis curve which a lot of reference have explained it $[10,11]$

In this paper, the loss phenomenology of the piezoelectric actuator (dielectric, mechanical, piezoelectric) is modeled and simulated using COMSOL Multiphysics software. Each loss

*E-mail address: rdnyjavier@cvsu.edu.ph

ISSN: 1791-2377 @ 2021 School of Science, IHU. All rights reserved.

doi:10.25103/jestr.144.19 is defined for specific factors: mechanical damping coefficient $\left(\mathrm{Q}_{\mathrm{M}}\right)$ for mechanical loss, dissipation factor $(\tan \delta)$ for dielectric loss and piezoelectric loss or coupling loss factor $(\tan \phi)$ for piezoelectric loss. The mechanical response, electrical impedance as well as the percentage loss for each loss is demonstrated over the frequency sweep of $1 \mathrm{kHz}$ to 1 $\mathrm{MHz}$. The mechanical response indicates the effect of each loss on the displacement of the PEA while the electrical impedance shows the behavior of the electrical resonances and the anti-resonances.

Mechanical quality factors are basically related to the losses and play a significant role for its study $[5,12]$. A higher mechanical quality factor increases the efficiency and reduces heat generation [7]. For PEAs, the resonances and antiresonances are considered as the maximum and minimum point of admittance which characterizes as the quality factor under resonance $\left(\mathrm{Q}_{\mathrm{a}}\right)$ and anti-resonance $\left(\mathrm{Q}_{\mathrm{b}}\right)$. In the study, the quality factors where obtained over the frequency sweep of the combined material loss configurations.

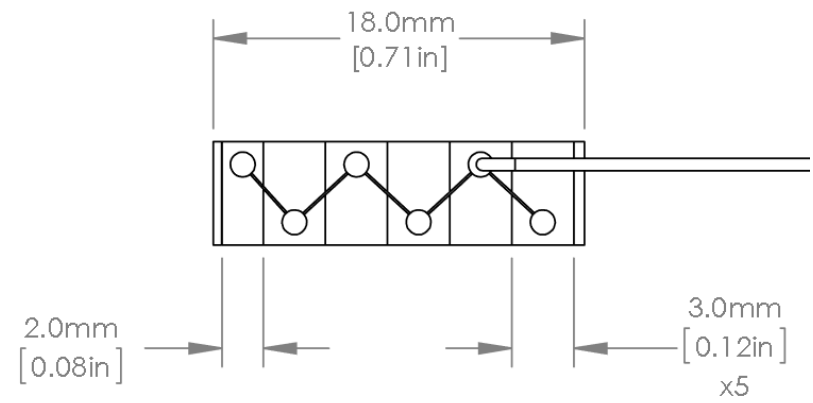

Fig. 1. Thors lab PK4FQ2 discrete piezoelectric rectangular stack.

\section{Methods}

2.1 Geometric Description

A discrete multilayer piezoelectric stack actuator with both flat ends was modelled in the simulation platform of 
COMSOL Multiphysics. The dimension specification was based on the Thors lab PK4FQP2 discrete piezoelectric rectangular stack. Different stack configurations were also implemented to test the difference in stack displacement and volumetric strain with variation to the actuator's layer thickness and overall dimension.

The geometry of the first actuator configuration is presented in Fig. 2. It also consisted of a piezoelectric stack of 6 single-chips piezoelectric. The chips are $3 \mathrm{~mm}$ thick and have surface area of $25 \mathrm{~mm}^{2}$. A piezoelectric stack actuator relies on the $d_{33}$ coupling mode and behaves linearly under constant voltage.

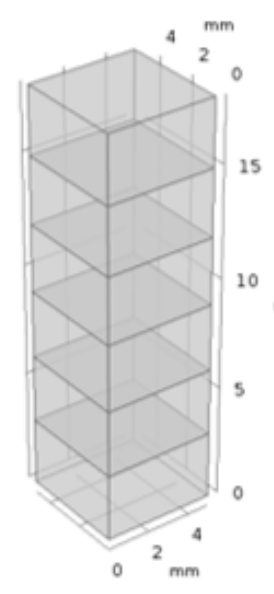

(a)

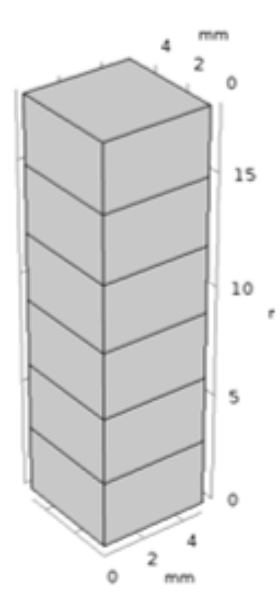

(b)

\subsection{Material Base Vector Coordinate System}

The piezoelectric material taken into consideration in the converse simulation is the Hard PZT Navy Type 1 (PZT-4). Each piezoelectric material has a poling vector $(Z)$ in which the $\varepsilon^{T}$, strain under applied constant electric field displaces along. In order to maximize the stack actuator displacement, alternation of Z+ polarized and Z- polarized PZT-4 material in each stack should be implemented.

\subsection{Applied Study and Multi-physics}

The actuator models were governed by the electrostatic and structural mechanics physics provided by the module available on the COMSOL simulation platform and were coupled by the piezoelectric device multi-physics module separately available on the program. The electric circuit module was also included to supply the actuator model with an external constant electric field supply and functions of sinusoidal and pulse wave oscillations.

\subsection{Dynamic Electrical Impedance Modeling}

The frequency domain was used to compute the response of the linear model to sweeping harmonic excitation produced by pulsating or sinusoidal for several scaling frequencies. The process itself is a simulated application of impedance analyser equipment.

The piezoelectric material is subjected to a frequency sweep, based on the basic operation of an impedance analyser, ranging from $1 \mathrm{kHz}$ to $1 \mathrm{MHz}$, with a step increase of $1 \mathrm{kHz}$. The boundary probe was used to measure the development of scalar-valued quantity (complex valued number) accumulation of the surface charge density from the dynamic frequency domain sweep simulation.

Fig. 2. Geometries of the different configurations.

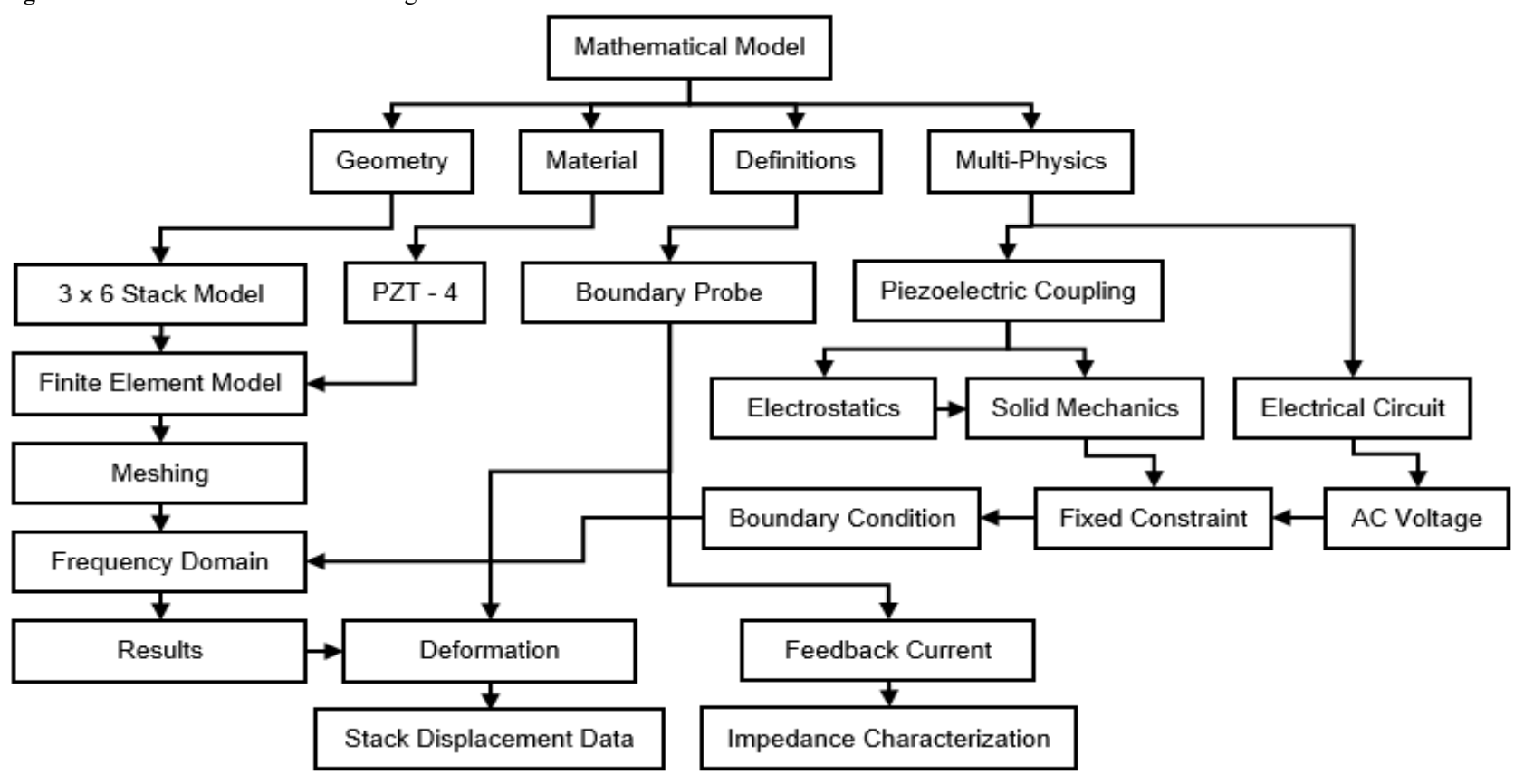

Fig. 3. Process of dynamic finite element modeling followed for simulation.

\subsection{Surface Charge Integration}

The expression given into the boundary probe was to compute the surface charge, $\boldsymbol{Q}(\boldsymbol{t})$ accumulated on the area, $\boldsymbol{m}^{2}$ of the electrode part of the actuator model. The expression given by: $\iint \frac{Q(t)}{m^{2}} d w d t=I(t)$

The integration of the charges by a sinusoidal function of time and by the out of plane thickness provides the measurement of current flown throughout the geometric model of the piezoelectric model.

\subsection{Complex Form of Current}

The loss tangent or the loss factor for the different material intensive losses are given as: 
Dielectric Loss: $\varepsilon^{T *}=\varepsilon^{T}\left(1-j \tan \delta^{\prime}\right)$

Compliance Loss: $s^{E *}=s^{E}\left(1-j \tan \phi^{\prime}\right)$

Coupling Loss: $d^{*}=d\left(1-j \tan \theta^{\prime}\right)$

Where $\boldsymbol{j}$ is the imaginary notation, $\boldsymbol{\varepsilon}^{\boldsymbol{T}}$ is the dielectric constant under constant stress $\boldsymbol{T}, \boldsymbol{s}^{\boldsymbol{E}}$ is the elastic compliance under constant electric field $\boldsymbol{E}, \boldsymbol{d}$ is the piezoelectric constant and $\boldsymbol{t a n} \boldsymbol{\delta}^{\prime}, \boldsymbol{t a n} \boldsymbol{\phi}^{\prime}$ and $\boldsymbol{\operatorname { t a n } \boldsymbol { \theta } ^ { \prime }}$ represent intensive dielectric, elastic and piezoelectric loss factors, respectively. Substituting the complex form of the different intensive parameter to the piezoelectric constitutive equation with the real parts as stored parameter and the imaginary parts as the loss parameter, the relation is given as:

$$
D=\varepsilon E+d \underline{\bar{X}}
$$

where the stress, $\underline{\bar{X}}$ is relate to compliance, $\boldsymbol{s}$ and strain, $\boldsymbol{x}$ by:

$\underline{\bar{X}}=\frac{s}{x}$

The constitutive relation becomes:

$$
D^{*}=\varepsilon^{T *} E+d^{*} \frac{s^{E *}}{x}
$$

$D^{*}=\varepsilon^{T}\left(1-j \tan \delta^{\prime}\right) E+d\left(1-j \tan \theta^{\prime}\right) \frac{s^{E}\left(1-j \tan \phi^{\prime}\right)}{x}$

Integrating the charge, $\boldsymbol{Q}^{*}(\boldsymbol{t})$ per area, $\boldsymbol{m}^{\mathbf{2}}$ from the electric displacement by the sinusoidal function of time and the out of plane thickness equates to the complex form of current, $\boldsymbol{I}$ with the different intensive losses produce by the sweeping frequency and is given as:

$$
\iint \frac{Q^{*}(t)}{m^{2}} d w d t=I^{*}=I(1-j \tan \alpha)
$$

\subsection{Mechanical Damping}

The mechanical damping subnode allows the modeling of mechanical losses in the piezoelectric material, either via using the loss factor material data for the stiffness, or in the form of the Rayleigh proportional damping. The damping type for the material chosen was the Isotropic loss factor. Although the material is anisotropic and therefore the losses inside the material should be anisotropic, the proponents had to assume that it is equally distributed among the vector components of the material because only the y displacement is measured by the tip deflection probe. The equation for the user defined mechanical damping, $\boldsymbol{\eta}_{\boldsymbol{S}}$ is inversely related to the Mechanical Quality factor, $\boldsymbol{Q}_{\boldsymbol{m}}$ of the material. The expression is given by:

$\tan \phi^{\prime}=\eta_{S}=\frac{1}{2 Q_{m}}$

\subsection{Dielectric Loss}

The dielectric Loss subnode allows the modeling electrical losses in the piezoelectric material. The losses can be prescribed either by using a loss factor for the electrical permittivity, or in the form of dielectric dispersion. The dielectric loss factor for the material was the loss factor for electrical permittivity under constant stress $\varepsilon^{T}$. The dielectric loss, or dissipation factor, $\boldsymbol{\eta}_{\varepsilon \underline{\bar{X}}}$ is the reciprocal of the electrical quality factor, $\boldsymbol{Q}_{\boldsymbol{E}}$ which represents the quality or durability of oscillation. The equation for the user defined dielectric loss factor is given by:

$\tan \delta^{\prime}=\eta_{\varepsilon^{\underline{X}}}=\frac{1}{Q_{E}}$

\subsection{Coupling Loss}

The coupling loss subnode allows the modeling of the losses in the piezoelectric coupling in the piezoelectric material either by using the loss factor material data for the coupling matrix or as Rayleigh proportional damping. The coupling loss factor for the material was the loss factor for piezoelectric constant $\boldsymbol{d}$. The loss factor is given by a $3 \times 6$ coupling matrix:

$$
\left[d^{\prime \prime}\right]=\left[\begin{array}{llllll}
d_{12}^{\prime \prime} & d_{12}^{\prime \prime} & d_{13}^{\prime \prime} & d_{14}^{\prime \prime} & d_{15}^{\prime \prime} & d_{16}^{\prime \prime} \\
d_{21}^{\prime \prime} & d_{22}^{\prime \prime} & d_{23}^{\prime \prime} & d_{24}^{\prime \prime} & d_{25}^{\prime \prime} & d_{26}^{\prime \prime} \\
d_{31}^{\prime \prime} & d_{32}^{\prime \prime} & d_{33}^{\prime \prime} & d_{34}^{\prime \prime} & d_{35}^{\prime \prime} & d_{36}^{\prime \prime}
\end{array}\right]
$$

\subsection{Post-processing of Data Results}

The tabular data simulated from the model under the static and frequency domain analysis, were presented as $1 \mathrm{D}$ group. $1 \mathrm{D}$ probe plot. The probe plot data were presented as a value of current that varies with frequency sweeping form $1 \mathrm{kHz}$ to $1 \mathrm{MHz}$. The phase lag plot data were presented as radians value of phase that varies with frequency sweeping form 1 $\mathrm{kHz}$ to $1 \mathrm{MHz}$. The phase lag between the lost, $\boldsymbol{U}_{\text {Lost }}$ and stored parameter, $\boldsymbol{U}_{\text {Stored }}$ is presented by the loss tangent equations given as:

Compliance Loss: $\tan \phi=\frac{U_{\text {Lost }}}{U_{\text {Stored }}}=\frac{\frac{1}{S^{E^{\prime \prime}}}}{\frac{1}{S^{E^{\prime}}}}=\frac{S^{E^{\prime}}}{S^{E^{\prime \prime}}}$

Dielectric Loss: $\tan \delta=\frac{\mathrm{U}_{\mathrm{L}}}{\mathrm{U}_{\mathrm{S}}}=\frac{\varepsilon \underline{\overline{\mathrm{x}}}^{\prime \prime}}{\varepsilon \underline{\mathrm{x}}^{\prime}}$

Coupling Loss: $\tan \theta=\frac{\mathrm{U}_{\mathrm{L}}}{\mathrm{U}_{\mathrm{S}}}=\frac{\mathrm{d}^{\overline{\mathrm{x}}^{\prime \prime}}}{\mathrm{d} \underline{\overline{\mathrm{x}}}^{\prime}}$

The phase lag $\boldsymbol{\alpha}$ is calculated by the expression:

$\alpha=\tan ^{-1} \frac{\text { real }(\text { bnd } 1)}{\operatorname{imag}(\text { bnd } 1)} * \frac{180}{\pi}$

The impedance plot data were presented as logarithmic scale value of impedance that varies with frequency sweeping form $1 \mathrm{kHz}$ to $1 \mathrm{MHz}$. The impedance value of the complex current is calculated by the expression given:

$Z=\frac{[V]}{(a b s(b n d 1))}$

where bnd 1 is the probe data, note that the resistivity of the PZT material is not defined and neglected in the analysis. We have to assume that the reactance is equal and in phase with the impedance data.

The Admittance plot data were presented as logarithmic scale value of admittance that varies with frequency sweeping form $1 \mathrm{kHz}$ to $1 \mathrm{MHz}$.

\section{Results and Discussion}

\subsection{Dynamic Frequency Modeling}

The piezoelectric actuator geometric model in the 2 dimension was subjected to harmonic perturbation of several scaling frequencies. The losses due to domain wall hysteresis are apparent in the frequency analysis and affect the 
displacement and impedance of the material at a given frequency.

The dynamic modeling also includes the resonance $f_{a}$ and anti-resonance $f_{b}$ frequency of the material in which the maximum and minimum value of the mechanical displacement and electric displacement is attained.

\subsection{Loss Phenomenology for Mechanical Damping Coefficient}

Stiffness is an example of an intensive property, which is defined as the extent to which an object resists deformation. The parameters for stiffness include the elastic compliance and elastic stiffnes or the young's modulus of a material. Losses on the structural domain are inherent because of the irreversible lost energy due to the lost parameter of the material stiffnes. The mechanical loss is inversely related to the mechanical factor of the material which denoted as $Q_{m}$.

\subsection{Mechanical response of mechanical loss model}

Based on the simulation results, the elastic loss parameter directly affects the displacement of the PZT ceramic.The mechanical displacement over the frequency sweep is shown in Fig. 4. Varying elastic loss factors shows different response to frequency sweep. The lower values of quality factor fails to follow through the resonance curve and instead falls through the anti-resonant path. These low value mechanical factors below 20 are identified as softer variant of PZT ceramics which in general are not suitable for actuation due to their high value compliance and loose form factor. Instead, their main applications are for transducer, benders, and energy harvester. On the other hand, quality factors above 20 which achieved mechanical resonance are classified as hard type PZT's, which are much stiffer and can withstand heavier loads and higher blocking force.

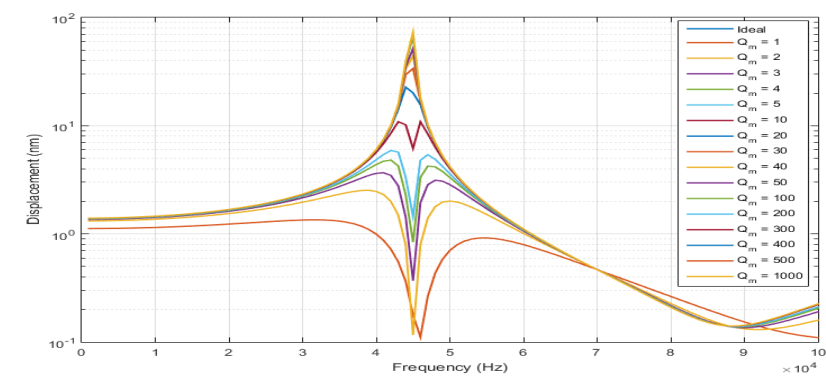

Fig. 4. Displacements of the PZT stack of different quality factor values..

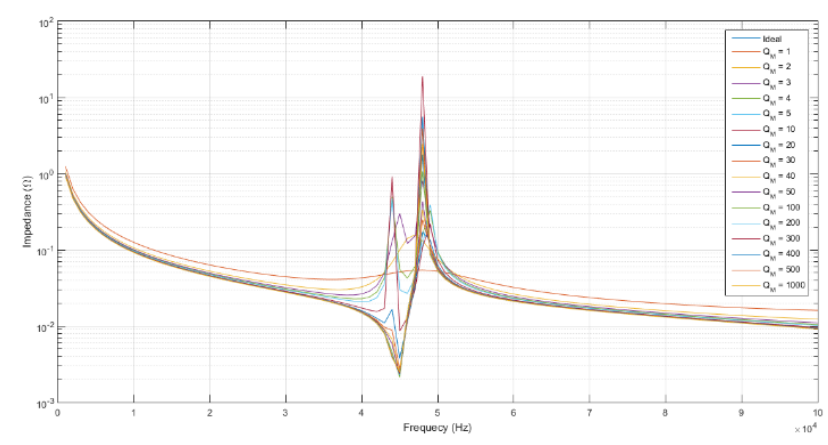

Fig. 5. Impedance data of mechanical damping loss factor.

\subsection{Electrical impedance of mechanical loss model.}

The mechanical loss factor also affects the coupled electrical domain of the piezoelectric material. The electrical impedance due to elastic compliance losses are shown in Fig. 5 and Fig. 6. The loss factor exhibits an initial anti-resonant peak just before reaching the resonant curve. Additionaly, the mechanical loss mainly varies the value of the impedance and admittance at the anti-resonance peak. The cut view of the impedance at the resonant and anti-resonant peak of the increasing mechanical loss factor to sweeping applied frequency was shown in Fig. 6.

The extended graph of the mechanical loss model impedance over the frequency sweep of $1 \mathrm{kHz}$ to $1 \mathrm{MHz}$ was shown in Fig. 5. This shows the variation of the impedance magnitude as well as the resonant and anti-resonant frequency of increasing mechanical loss factor.

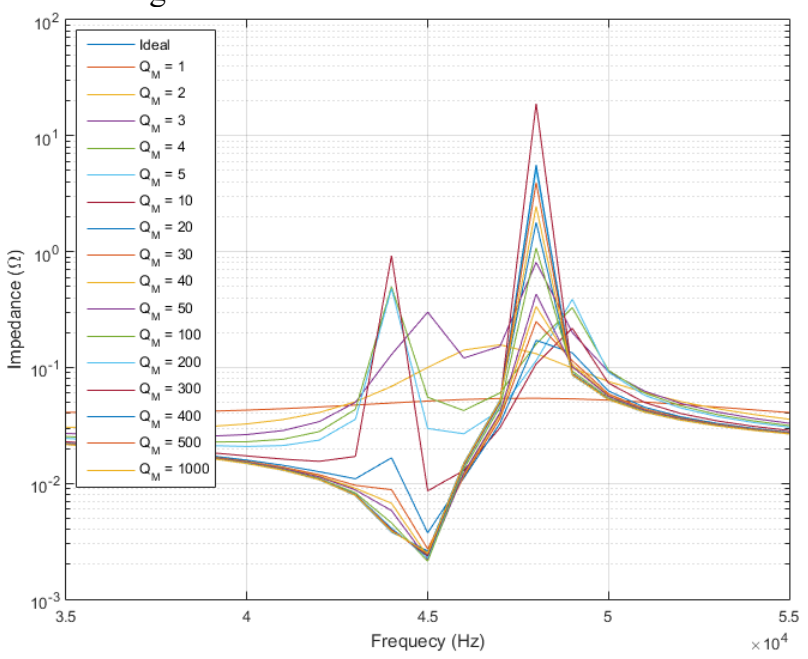

Fig. 6. Mechanical loss impedance at the $\boldsymbol{f}_{\boldsymbol{a}}$ and $\boldsymbol{f}_{\boldsymbol{b}}$ peak curve

\subsection{Percentage loss of mechanical damping coefficient}

The percentage loss over the frequency sweep for the mechanical damping coefficient loss factor of the 6-layer stack model is shown in Fig. 7. The percentage loss values for the scientific frequency range values for the resonant and antiresonant frequency is shown in Tab. 1.



Fig. 7. Percent loss vs frequency of the mechanical loss factor

\subsection{Loss Phenomenology for Dielectric Loss}

The dielectric loss, also termed as dissipation factors, is evident to dielectric materials such as capacitors. Piezoelectric material behaves and is basically a capacitive element. This material loss occurs due to the lagging of the polarization behind the applied electric by the phase angle $\tan \delta$ which constitutes to the loss factor of the dielectric permittivity $\varepsilon$. 
Table 1. Percentage Loss of Mechanical Damping Loss Factor

\begin{tabular}{|c|c|c|c|c|c|}
\hline \multicolumn{6}{|c|}{ QUALITY FACTOR, $Q_{m}$} \\
\hline Frequency & 1 & 2 & 3 & 4 & 5 \\
\hline Off Resonance & 18.7650 & 10.3525 & 7.0366 & 5.3142 & 4.2646 \\
\hline Resonance & 32.8259 & 25.5419 & 21.9881 & 20.8912 & 20.7922 \\
\hline Anti-Resonance & 51.6164 & 81.0613 & 105.1230 & 85.4404 & 85.8435 \\
\hline Lower $3 \mathbf{d b} \mathbf{f}_{\mathbf{r}}$ & 31.3762 & 23.2110 & 19.2154 & 17.5519 & 17.4114 \\
\hline Upper 3db f $\mathbf{r}_{\mathrm{r}}$ & 34.4238 & 28.4400 & 25.7058 & 25.6917 & 28.4394 \\
\hline \multicolumn{6}{|c|}{ QUALITY FACTOR, $\mathbf{Q}_{\mathrm{m}}$} \\
\hline Frequency & 10 & 20 & 30 & 40 & $\mathbf{5 0}$ \\
\hline Off Resonance & 2.1415 & 1.0718 & 0.7148 & 0.5361 & 0.4289 \\
\hline Resonance & 23.7922 & 253.5170 & 137.6312 & 96.9140 & 75.3986 \\
\hline Anti-Resonance & 66.2727 & 446.4101 & 475.8433 & 451.7016 & 412.0586 \\
\hline Lower $3 d b f_{r}$ & 15.9905 & 64.5194 & 45.6010 & 34.9377 & 28.2314 \\
\hline Upper 3db f & 41.2327 & 76.7471 & 47.8606 & 35.1010 & 27.7957 \\
\hline \multicolumn{6}{|c|}{ QUALITY FACTOR, $\mathbf{Q}_{\mathrm{m}}$} \\
\hline Frequency & 100 & 200 & 300 & 400 & 500 \\
\hline Off Resonance & 0.2145 & 0.1072 & 0.0715 & 0.0536 & 0.0429 \\
\hline Resonance & 36.3644 & 18.0228 & 11.9955 & 8.9916 & 7.1913 \\
\hline Anti-Resonance & 253.447 & 134.4629 & 90.6672 & 68.2741 & 54.722 \\
\hline Lower 3db $f_{r}$ & 14.3083 & 7.1786 & 4.7887 & 3.5923 & 2.8741 \\
\hline Upper 3db $\mathbf{f}_{\mathbf{r}}$ & 13.7122 & 6.8333 & 4.5527 & 3.4138 & 2.7308 \\
\hline \multicolumn{6}{|c|}{ QUALITY FACTOR, $\mathbf{Q}_{\mathrm{m}}$} \\
\hline Frequency & \multicolumn{5}{|c|}{1000} \\
\hline Off Resonance & \multicolumn{5}{|c|}{0.0214} \\
\hline Resonance & \multicolumn{5}{|c|}{3.5944} \\
\hline Anti-Resonance & \multicolumn{5}{|c|}{27.4292} \\
\hline Lower $3 d b f_{r}$ & \multicolumn{5}{|c|}{1.4373} \\
\hline Upper 3db $f_{r}$ & \multicolumn{5}{|c|}{1.3652} \\
\hline
\end{tabular}

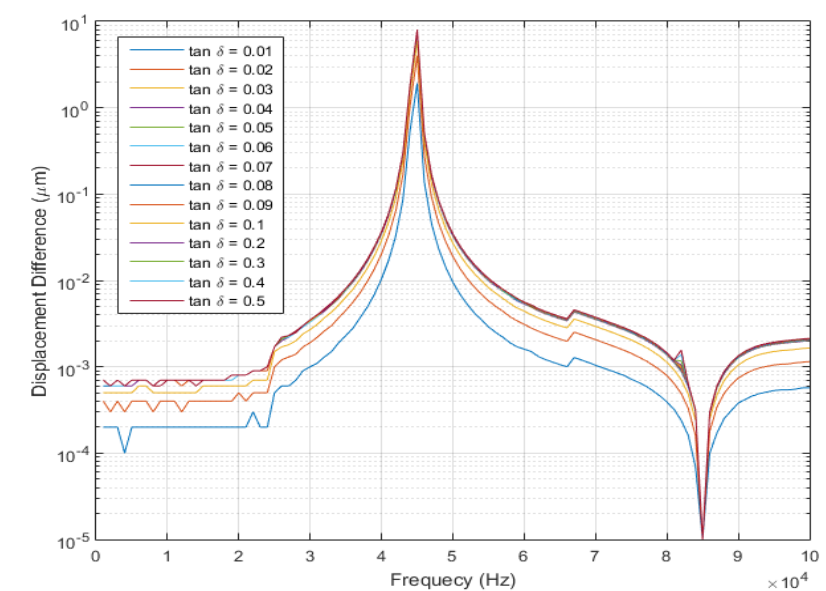

Fig. 8. Displacement vs. frequency line graph of the dielectric loss model.

\subsection{Mechanical response of the dielectric loss}

Unlike the mechanical damping loss factor, the dielectric loss virtually has very little effect on the mechanical displacement of the piezoelectric stack model. The displacement over the frequency sweep is shown in Fig. 8.

\subsection{Electrical impedance of the dielectric loss}

Like the mechanical loss, the same resonance frequency is achieved by the simulation around $48 \mathrm{kHz}$. This implies that the resonant frequency is an extensive property of the PZT material and is invariant to the losses. The dielectric loss also majorly affects magnitude of the impedance of the antiresonance peak and the frequency in which the anti-resonance is achieved. The impedance data of the resonance and the antiresonance peak is shown in Fig. 9 and 10.

The extended graph of the dielectric loss model impedance over the frequency sweep of $1 \mathrm{kHz}$ to $1 \mathrm{MHz}$ was shown in Fig. 9. This shows the variation of the impedance magnitude as well as the resonant and anti-resonant frequency of increasing dielectric loss factor.The cut view of the impedance at resonant and anti -resonant peak of the increasing dielectric loss factor to sweeping applied frequency was shown in Fig. 9.

\subsection{Percentage loss for dielectric loss}

The percentage loss over the frequency sweep for the dielectric loss factor of the 6-layer stack model is shown in Fig. 11. The percentage loss values for the scientific frequency range values for the resonant and anti-resonant frequency is shown in Tab. 2 .

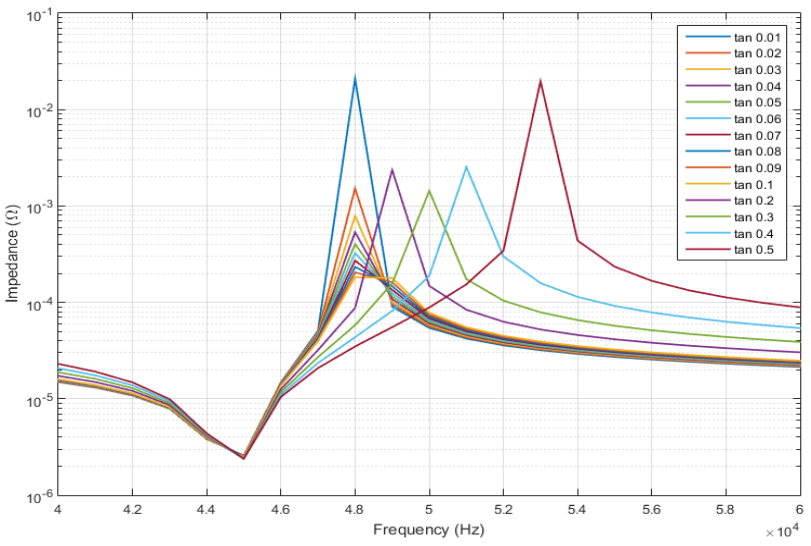

Fig. 9. Dielectric loss impedance at the $\boldsymbol{f}_{\boldsymbol{a}}$ and $\boldsymbol{f}_{\boldsymbol{b}}$ peak curve. 
Table 2. Percentage Loss of Dielectric Loss Factor

\begin{tabular}{c|c|c|c|c|c}
\hline \multicolumn{7}{c}{ DIELECTRIC LOSS (TAN $\boldsymbol{~}$ ) } \\
\hline Frequency & $\mathbf{0 . 0 1}$ & $\mathbf{0 . 0 2}$ & $\mathbf{0 . 0 3}$ & $\mathbf{0 . 0 4}$ & $\mathbf{0 . 5}$ \\
\hline Off Resonance & 1.119 & 2.239 & 3.359 & 4.478 & 5.598 \\
Resonance & 0.455 & 0.909 & 1.364 & 1.819 & 2.273 \\
Anti-resonance & 108.5 & 216.6 & 323.6 & 429.2 & 532.8 \\
Lower 3dB $\omega_{a 1}$ & 0.652 & 1.304 & 1.956 & 2.608 & 3.259 \\
Upper 3dB $\omega_{a 2}$ & 0.647 & 1.294 & 1.939 & 2.582 & 3.224 \\
\hline Frequency & $\mathbf{0 . 0 6}$ & $\mathbf{0 . 0 7}$ & $\mathbf{0 . 0 8}$ & $\mathbf{0 . 0 9}$ & $\mathbf{0 . 1}$ \\
\hline Off Resonance & 6.718 & 7.837 & 8.957 & 10.08 & 11.19 \\
Resonance & 2.727 & 3.181 & 3.634 & 4.087 & 4.539 \\
Anti-resonance & 634.1 & 732.7 & 828.3 & 920.6 & 1009 \\
Lower 3dB $\omega_{a 1}$ & 3.911 & 4.563 & 5.215 & 5.866 & 6.517 \\
Upper 3dB $\omega_{a 2}$ & 3.862 & 4.496 & 5.126 & 5.752 & 6.372 \\
\hline Frequency & $\mathbf{0 . 1}$ & $\mathbf{0 . 2}$ & $\mathbf{0 . 3}$ & $\mathbf{0 . 4}$ & $\mathbf{0 . 5}$ \\
\hline Off Resonance & 11.19 & 22.39 & 33.59 & 44.78 & 17.68 \\
Resonance & 4.539 & 9.021 & 13.41 & 2221 & 21.84 \\
Anti-resonance & 1009 & 1685 & 2039 & 25.94 & 2338 \\
Lower 3dB $\omega_{a 1}$ & 6.517 & 13.02 & 19.49 & 21.11 & 32.35 \\
Upper 3dB $\omega_{a 2}$ & 6.372 & 12.19 & 17.13 & 24.24 \\
\hline
\end{tabular}

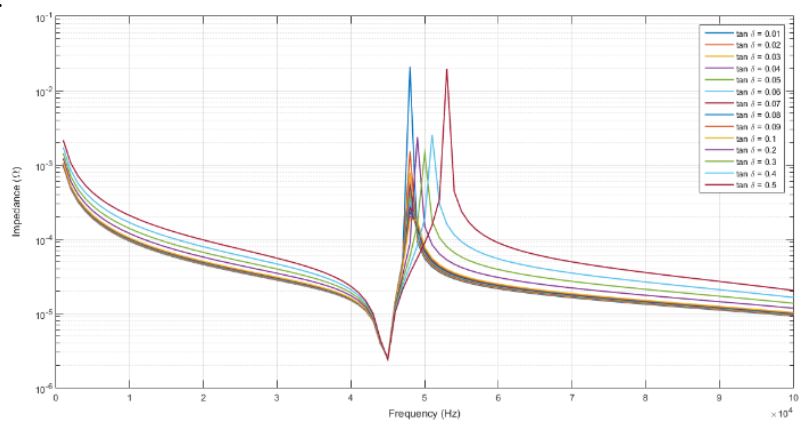

Fig. 10. Impedance graph of the dielectric loss factor.

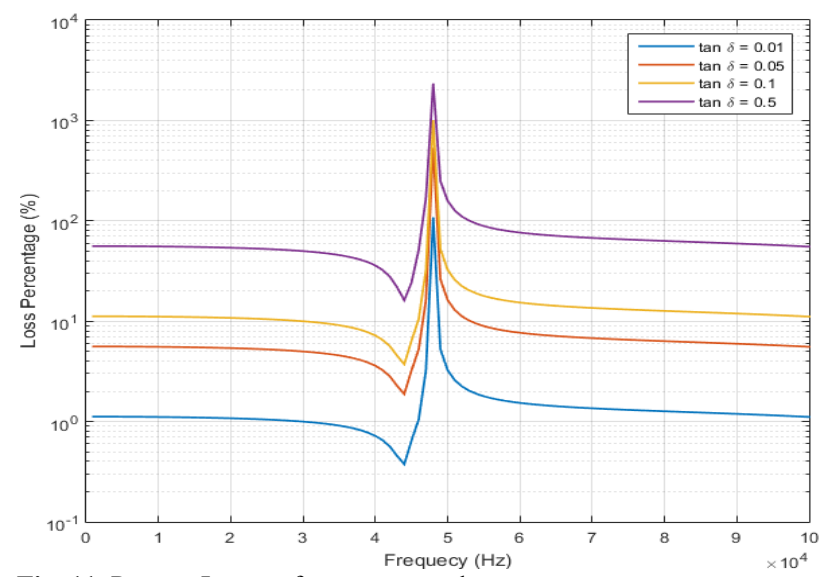

\subsection{Loss Phenomenology for Coupling Loss}

Coupling loss is also termed as the loss factor of the piezoelectric constant. It is defined as the loss factor of the electromechanical coupling. This material loss is due to the lagging of the polarization behind the stress applied or the lagging of strain behind the applied electric field by the phase angle $\tan \theta$ which constitutes $\mathrm{x}$ to the loss factor of the piezoelectric charge constant $d$.

\subsection{Mechanical response of coupling loss model}

The logarithmic scale nanometer displacement over the frequency sweep of $1 \mathrm{kHz}$ to $1 \mathrm{MHz}$ for the increasing value of coupling loss factor is shown in Fig. 12.

\subsection{Electrical impedance of coupling loss}

The extended graph of the coupling loss model impedance over the frequency sweep of $1 \mathrm{kHz}$ to $1 \mathrm{MHz}$ was shown in Fig. 13. This shows the variation of the impedance magnitude as well as the resonant and anti-resonant frequency to increasing coupling loss factor.

The cut view of the impedance at resonant and anti resonant peak of the increasing coupling loss factor to sweeping applied frequency was shown in Fig. 14.

Table 3. Percentage loss of coupling loss factor

\begin{tabular}{c|c|c|c|c|c}
\hline \multicolumn{7}{c}{ DIELECTRIC LOSS (TAN $\boldsymbol{\theta}$ ) } \\
\hline Frequency & $\mathbf{0 . 0 0 1}$ & $\mathbf{0 . 0 0 2}$ & $\mathbf{0 . 0 0 3}$ & $\mathbf{0 . 0 0 4}$ & $\mathbf{0 . 0 0 5}$ \\
\hline Off Resonance & 0.024 & 0.048 & 0.072 & 0.096 & 0.119 \\
Resonance & 0.109 & 0.218 & 0.327 & 0.436 & 0.545 \\
Anti-resonance & 21.52 & 43.03 & 64.5 & 85.91 & 107.3 \\
Lower 3dB $\omega_{a 1}$ & 0.067 & 0.139 & 0.209 & 0.278 & 0.348 \\
Upper 3dB $\omega_{a 2}$ & 0.329 & 0.659 & 0.988 & 1.318 & 1.647 \\
\hline Frequency & $\mathbf{0 . 0 1}$ & $\mathbf{0 . 0 2}$ & $\mathbf{0 . 0 3}$ & $\mathbf{0 . 0 4}$ & $\mathbf{0 . 0 5}$ \\
\hline Off Resonance & 0.144 & 0.167 & 0.191 & 0.215 & 0.239 \\
Resonance & 0.654 & 0.763 & 0.872 & 0.981 & 0.109 \\
Anti-resonance & 128.5 & 149.7 & 170.7 & 191.6 & 212.3
\end{tabular}


Mark Christian P. Espinosa, Paola N. Calub, Rodney B. Javier and Sheryl Dinglasan Fenol/

Journal of Engineering Science and Technology Review 14 (4) (2021) 153 - 160

\begin{tabular}{c|c|c|c|c|c|} 
Lower $3 \mathrm{~dB} \omega_{a 1}$ & 0.418 & 0.487 & 0.557 & 0.626 & 0.696 \\
Upper 3dB $\omega_{a 2}$ & 1.977 & 2.306 & 2.636 & 2.966 & $\mathbf{0 . 4}$ \\
\hline Frequency & $\mathbf{0 . 1}$ & $\mathbf{0 . 2}$ & $\mathbf{0 . 3}$ & 0.956 & 1.195 \\
\hline Off Resonance & 0.239 & 0.478 & 0.717 & 4.37 & 5.46 \\
Resonance & 1.09 & 2.18 & 3.27 & 704.8 & 799.5 \\
Anti-resonance & 212.3 & 407.9 & 574.2 & 2.786 & 3.484 \\
Lower 3dB $\omega_{a 1}$ & 0.696 & 1.392 & 2.089 & 13.23 & 16.57 \\
Upper 3dB $\omega_{a 2}$ & 3.295 & 6.595 & 9.905 & \\
\hline
\end{tabular}

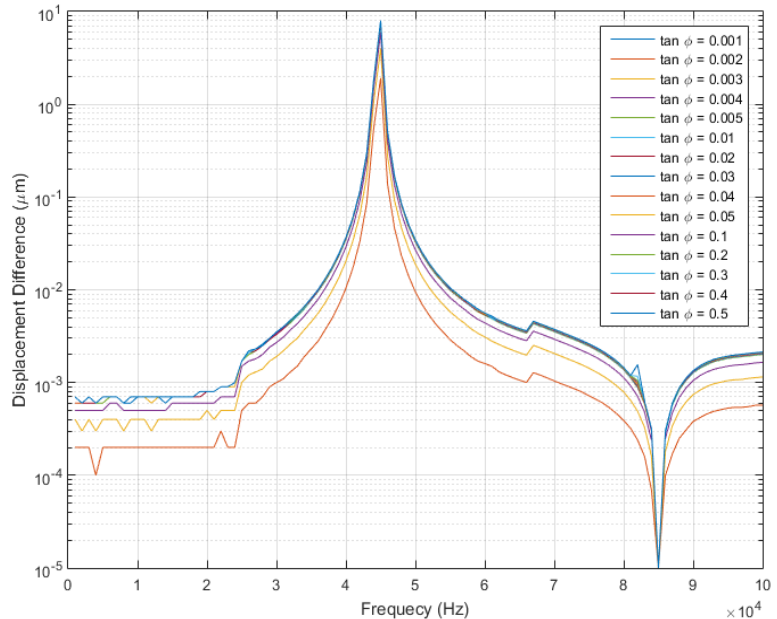

Fig. 12. Displacement vs. frequency line graph of the coupling loss model

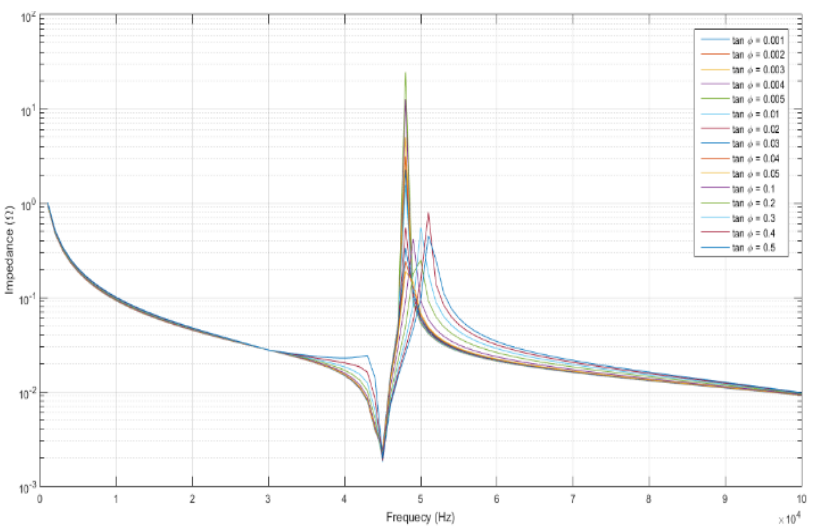

Fig. 13. Impedance data of coupling loss factor.

\subsection{Percentage loss for coupling loss}

The percentage loss over the frequency sweep for the coupling loss factor of the 6-layer stack model is shown in Fig. 15. The percentage loss values for the scientific frequency range values for the resonant and anti-resonant frequency is shown in Tab. 3

\subsection{Piezoelectric Quality Factor}

The quality factor is the figure of merit for the material in terms of efficiency or heat generation, and it is closely related to loss factors (Zhuang, 2011). In the simulation, the quality factor $Q_{a}$ or $Q_{b}$ can be obtained around the resonance or antiresonance peak of the admittance curve.

The admittance over the frequency sweep of the combined material loss configurations was shown in Fig. 16. The loss configurations are ranges of increasing values of loss factor for each loss for mechanical, dielectric and coupling.

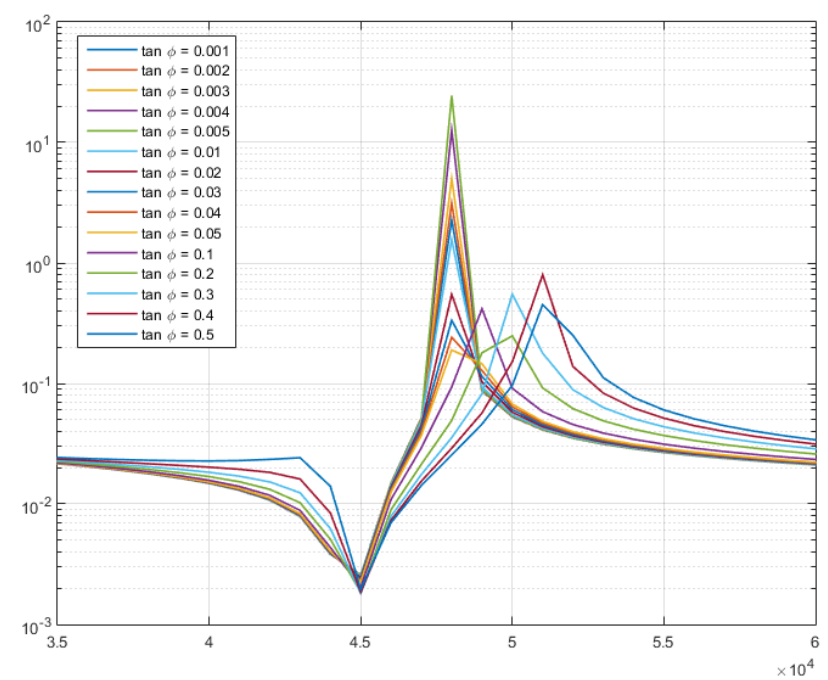

Fig. 14. Coupling loss impedance at the $\boldsymbol{f}_{\boldsymbol{a}}$ and $\boldsymbol{f}_{\boldsymbol{b}}$ peak curve.

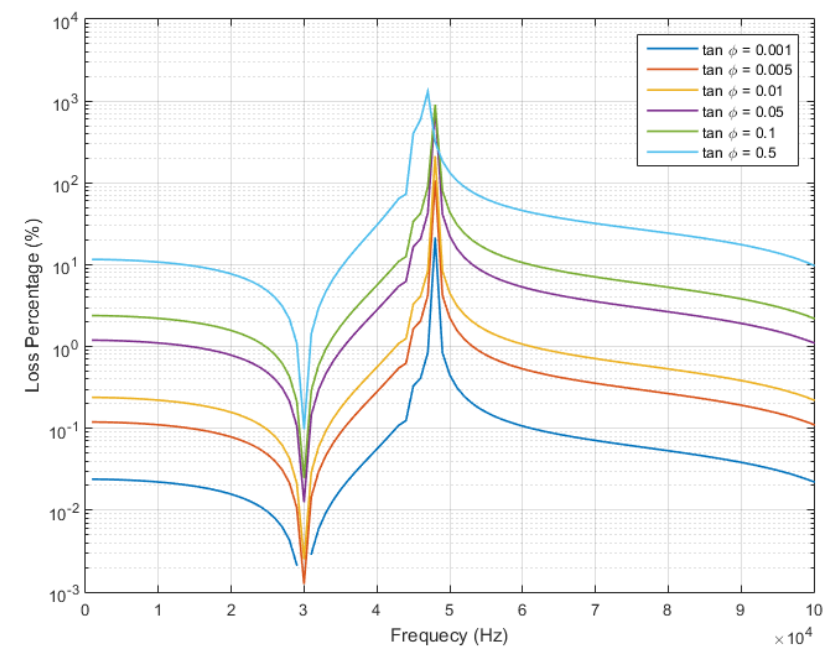

Fig. 15. Percent loss vs. frequency graph.

\section{Conclusion}

The mechanical damping coefficient was the parameter used to define the mechanical loss. Based on the simulation, the mechanical loss parameter directly affects the displacement of the PZT ceramic. Varying the elastic loss factors shows different responses on the frequency sweep. Lower values of quality factor failed to follow the resonance curve and instead fell on the anti-resonant path. These low value mechanical factors below 20 were identified as softer variant of PZT ceramics which in general were not suitable for actuation due to their high value compliance and loose form factor. The mechanical loss factor also affects the coupled electrical domain of the PZT's. This varies the value of the impedance and admittance at the anti-resonance peak. Furthermore, the loss percentage varies at different frequencies, which implies 
that the elastic loss factor is different for each given frequency.

The dissipation factor was used to define the dielectric loss. Unlike the mechanical damping loss factor, the dielectric loss has virtually little effect on the mechanical displacement. However, the same resonance frequency was achieved by the simulation around $48 \mathrm{kHz}$. This implies that the resonant frequency is an extensive property of the PZT material and is invariant to the losses. The dielectric loss also majorly affects magnitude of the impedance of the anti-resonance peak and the frequency in which the anti-resonance is achieved. The loss factor on the resonant peak is minimal and negligible but there is a great effect on anti-resonance peak. The percentage loss linearly increases by 1.11 to $1.12 \%$.

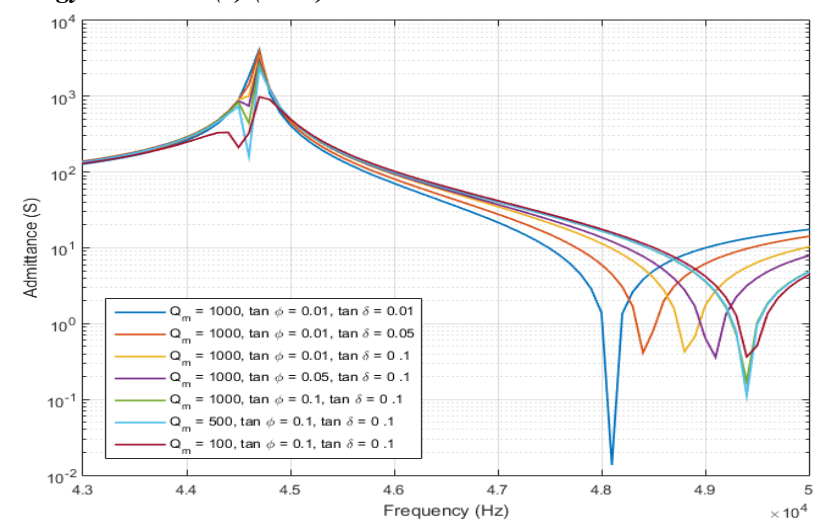

Fig. 16. Resonance and Anti-resonance admittances of the loss configurations.

Table 4. Piezoelectric Quality Factor of Good to Bad Values of Material Loss

\begin{tabular}{c|c|c|c|c}
\hline \multicolumn{2}{|c|}{ MATERIAL LOSS } & \multicolumn{2}{c}{ QUALITY FACTOR } \\
\hline $\boldsymbol{Q}_{\boldsymbol{m}}$ & $\tan \boldsymbol{\phi}$ & $\tan \boldsymbol{\delta}$ & $\boldsymbol{Q}_{\boldsymbol{a}}$ & $\boldsymbol{Q}_{\boldsymbol{b}}$ \\
\hline $\mathbf{1 0 0 0}$ & $\mathbf{0 . 0 1}$ & $\mathbf{0 . 0 1}$ & 698.44 & 3200 \\
$\mathbf{1 0 0 0}$ & $\mathbf{0 . 0 1}$ & $\mathbf{0 . 0 5}$ & 698.43 & 1452.94 \\
$\mathbf{1 0 0 0}$ & $\mathbf{0 . 0 1}$ & $\mathbf{0 . 1}$ & 677.27 & 6422.73 \\
$\mathbf{1 0 0 0}$ & $\mathbf{0 . 0 5}$ & $\mathbf{0 . 1}$ & 677.27 & 575.33 \\
$\mathbf{1 0 0 0}$ & $\mathbf{0 . 1}$ & $\mathbf{0 . 1}$ & 596.93 \\
$\mathbf{5 0 0}$ & $\mathbf{0 . 1}$ & $\mathbf{0 . 1}$ & 201.35 & 478.43 \\
$\mathbf{1 0 0}$ & $\mathbf{0 . 1}$ & $\mathbf{0 . 1}$ & & 382.95 \\
\hline
\end{tabular}

Lastly, piezoelectric loss factor, also called the coupling loss, was used to define the piezoelectric loss. The coupling loss showed identical mechanical response with the dielectric loss model. The magnitude of impedance varied at increasing loss factor. The mechanical resonant and anti-resonant frequency is the same for all values of coupling loss. The coupling loss also exhibited both effects of mechanical and dielectric loss in terms of electrical properties. Similarly, the loss factor at the off-resonance range was minimal and the resonant frequency was the same for all values of coupling loss. The loss percentage for the coupling loss model scales nonlinearly to increasing loss factor and input frequency.
From the simulation, the combination of material loss which has higher mechanical loss parameter, lower dielectric loss and lower piezoelectric loss factor has a higher the quality factor under resonance $\left(\mathrm{Q}_{\mathrm{a}}\right)$ and anti-resonance $\left(\mathrm{Q}_{\mathbf{b}}\right)$. It decreases as the mechanical loss decreases and the dielectric and piezoelectric loss factor increases.

This is an Open Access article distributed under the terms of the Creative Commons Attribution License.

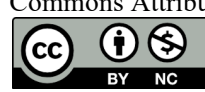

\section{References}

1. W. Li, and X. Chen, "Compensation of hysteresis in piezoelectric actuators without dynamics modeling", Sensors and Actuators A: Physical, 2013, Vol. 199, pp. 89-97.

2. Z. Chi, and Q. Xu, "Recent advances in the control of piezoelectric actuators", International Joural of Advanced Robotic Systems, 2014, Vol. 11, No. 182, pp. 1-11.

3. Y. Zhuang, "Loss phenomonology and the methodology to derive loss factors in piezoeletric ceramics". Pennsylvania, USA: Pennsylvania State University, 2011.

4. L. Cheng, W. Liu, Z. G. Hou, J. Yu, and M. Tan, "Neural network based nonlinear model predictive control for piezoelectric actuators", IEEE Transactiond on Industrial Electronics, 2015, Vol. 62, No. 12, pp. 7717 - 7727.

5. M. Zhang, and D. Damjanovic, "A quasi-rayleigh model for modeling hysteresis of piezoelectric actuators", Smart Materials and Structures, 2020, Vol. 29, pp. 1-16.

6. Y. Zhuang, S. O. Ural, and K. Uchino, "Methodology for characterizing loss factors of piezoelectric ceramics", Ferroelectrics, 2014, Vol. 470, pp. 260-271.

7. K. Uchino, Y. Zhuang, and S. O. Ural, " Loss determination methodology for a piezoelectric ceramic: new phenomenological theory and experimental proposals", Journal of Advanced Dielectrics, 2011, Vol. 1 No. 1, pp. 17-31.

8. K. Uchino, and S. Hirose, "Loss mechanisms in piezoelectrics: how to measure different losses separately", IEEE Transactions on Ultrasonics, Ferroelectrics and Frequency Control, 2001, Vol. 48, No. 1, pp. 307-321.

9. J. Peng, and X. Chen, "A survey of modeling and control of piezoelectric actuators", Modern Mechanical Engineering, 2013, Vol. 3, pp. 1-20.

10. R. Pomirleanu, and V. Giurgiutiu, "High-field chracterization of piezoelectric and magnetostrictive actuators", Journal of Intelligent Material Systems and Structures, 2004, Vol. 15, pp. 161-180.

11. R. Kohler, and S. Rinderknecht, "A phenomenological approach to temperature dependent piezo stack actuator modeling", Sensors and Actuators A: Physical, Vol. 200, pp. 123-132.

12. A. V. Mezheritsky, "Elastic, dielectric and piezoelectric losses in piezoceramics: how it works all together", IEEE Transactions on Ultrasonics, Ferroelectrics and Frequency Control, 2004, Vol. 51, No. 6, pp. 695-707. 